\title{
Stochastic Quantization of Scalar Fields in Einstein and Rindler Spacetime
}

\author{
G. Menezes ${ }^{1}$ and N. F. Svaiter ${ }^{2}$ \\ Centro Brasileiro de Pesquisas Físicas-CBPF, \\ Rua Dr. Xavier Sigaud 150, \\ Rio de Janeiro, RJ, 22290-180, Brazil
}

\begin{abstract}
We consider the stochastic quantization method for scalar fields defined in a curved manifold and also in a flat space-time with event horizon. The two-point function associated to a massive self-interacting scalar field is evaluated, up to the first order level in the coupling constant $\lambda$, for the case of an Einstein and also a Rindler Euclidean metric, respectively. Its value for the asymptotic limit of the Markov parameter $\tau \rightarrow \infty$ is exhibited. The divergences therein are taken care of by employing a covariant stochastic regularization, where all the symmetries of the original theory are preserved.
\end{abstract}

PACS numbers: $03.70+\mathrm{k}, 04.62 .+\mathrm{v}$

\footnotetext{
${ }^{1}$ e-mail: gsm@cbpf.br

${ }^{2}$ e-mail: nfuxsvai@cbpf.br
} 


\section{Introduction}

In the last century Parisi and $\mathrm{Wu}$ introduced the stochastic quantization method [1]. The main idea of the stochastic quantization is that a $d$-dimensional quantum system is equivalent to a $(d+1)$-dimensional classical system including fluctuations effects into considerations. Some of the most important papers in the subject can be found in Ref. [2]. A brief introduction to the stochastic quantization can be found in the Refs. [3] and [4] and a complete review of the stochastic quantization is given in the Ref. [5].

This program of stochastic quantization and the stochastic regularization was carried out for generic fields defined in flat, Euclidean manifolds. It is important to observe that, since the stochastic regularization is not an action regularization, this method preserves all the symmetries of the theory under study. In the development of this program some authors applied this method to linearized Euclidean gravity and also non-linearized gravity [6] [7] [8] [9] [10]. It is worth pointing out here that we cannot expect that the method will improve the perburbative nonrenormalizability of quantum gravity. Working along such lines, we may observe that the study of a situation which lies between these two extremes is missing. Therefore, before the attempt to implement the program in non-renormalizable models, a consistent logical step is to discuss an intermediate situation between fields in flat spacetime and quantum gravity, i.e., the semiclassical theory, which corresponds to the one-loop approximation to the totally quantized theory. It is tempting to think that many unsolved problems, as the loss of information in black hole formation and the entropy of black holes, can gain new insights by introducing the stochastic quantization.

Although there are some papers discussing the stochastic quantization in Minkowski spacetime [11] [12], the stochastic quantization was originally introduced as an alternative quantization method for fields defined in Euclidean space. Therefore our aim in this article is to discuss the stochastic quantization of scalar fields defined in a static curved manifold without event horizon and also in a flat manifold with event horizon i.e., to analytically continue metrics that generate real actions. We would like to stress that the stochastic quantization is quite different from the other quantization methods, therefore it can reveal new structural elements of a theory which so far have gone unnoticed.

The method of stochastic quantization in flat spacetime with trivial topology can be summarized by the following steps. First, starting from a field defined in Minkowski spacetime, after analytic continuation to imaginary time, the Euclidean counterpart, i.e., the field defined in an Euclidean space, is obtained. Second, it is introduced a monotonically crescent Markov parameter, called in the literature "fictitious time" and also a random noise field $\eta(\tau, x)$, which simulates the coupling between the classical system and a heat reservoir. It is assumed that the fields defined at the beginning in a $d$-dimensional Euclidean space also depends on the Markov parameter, there- 
fore the field and a random noise field are defined in a $(d+1)$-dimensional manifold. One starts with the system out of equilibrium at an arbitrary initial state. It is then forced into equilibrium assuming that its evolution is governed by a Markovian Langevin equation with a white random noise field [13] [14] [15]. In fact, this evolution is described by a process which is stationary, Gaussian and Markovian. Finally, the $n$-point correlation functions of the theory in the $(d+1)$ dimensional space are defined by performing averages over the random noise field with a Gaussian distribution, that is, performing the stochastic averages $\left\langle\varphi\left(\tau_{1}, x_{1}\right) \varphi\left(\tau_{2}, x_{2}\right) \ldots \varphi\left(\tau_{n}, x_{n}\right)\right\rangle_{\eta}$. The $n$ point Schwinger functions of the Euclidean $d$-dimensional theory are obtained evaluating these $n$-point stochastic averages $\left\langle\varphi\left(\tau_{1}, x_{1}\right) \varphi\left(\tau_{2}, x_{2}\right) \ldots \varphi\left(\tau_{n}, x_{n}\right)\right\rangle_{\eta}$ when the Markov parameter goes to infinity $(\tau \rightarrow \infty)$, and the equilibrium is reached. This can be proved in different ways for the particular case of Euclidean scalar field theory. One can use, for instance, the Fokker-Planck equation [16] [17] associated with the equations describing the stochastic dynamic of the system. A diagrammatical technique [18] has also been used to prove such equivalence.

The original method proposed by Parisi and $\mathrm{Wu}$ was extended to include theories with fermions [19] [20] [21]. The first question that appears in this context is if make sense the Brownian problem with anticommutating numbers. It can be shown that, for massless fermionic fields, there will not be a convergence factor after integrating the Markovian Langevin equation. Therefore the equilibrium is not reached. One way of avoiding this problem is to introduce a kernel in the Langevin equation describing the evolution of two Grassmannian fields.

Usually, the Parisi-Wu scheme of quantization applied to bosonic and fermionic fields converges towards non-regularized theories. In order to obtain regularized stochastic diagrams, the original stochastic process proposed by Parisi and $\mathrm{Wu}$ must be modified. One way is to replace the Markovian process by a non-Markovian one [22] [23]. This can be done introducing a colored noise in the Einstein relations [24] [25] [26] [27]. Recently, the stochastic quantization of a topological Chern-Simons theory and the self-interacting $\lambda \varphi^{4}$ theory was investigated by the authors, using the Parisi-Wu scheme with a non-Markovian Langevin equation [28] [29].

Motivated by the problems that appear when one tries to implement this regularization scheme in non-abelian gauge theories with Zwanziger's gauge fixing [30] [31] [32], Bern et al [33] derived a truly non-perturbative regularization procedure in $Q C D$, still in the Markovian scenario. They introduced a modified Langevin equation with a regulator multiplying the noise. Therefore, the original Einstein relations and consequently the noise probability distribution are maintained. This continuum regularization program was very successful in the invariant non-perturbative regularization of all quantum field theories [34] [35] [36], including gauge theory, gauge theory with fermions and gravity. In few words, the idea of any stochastic regularization is to start from an interacting theory, then construct Langevin tree-graphs, where each leg of which ends in a regularized noise factor. Since it is possible to obtain the loops of the theory by contracting the noise factors, one ends up with a theory where every closed loop contains at least some power of the 
regulator. With this modification, one can show that the system converges towards a regularized theory. The next step to construct a finite theory is to use for example a minimal-subtraction scheme in which the ultraviolet divergent contributions are eliminated.

The aim of this paper is to implement the stochastic quantization for the self-interacting $\lambda \varphi^{4}$ theory in a Riemannian manifold and also in a flat manifold with event horizon, being more specific, the Einstein [37] and the Rindler spacetime [38] [39] [40] [41], where positive-definite forms can be obtained which generate real actions in the Parisi-Wu Langevin equation. The only article that has some overlap with our work is the Huang paper [42]. This author have used the stochastic quantization to find the regularized stress-energy tensor associated to a scalar field in an inhomogeneous spacetime. We would like to stress that although Brownian motion on a manifold has been studied by the mathematicians, there are a few papers studying Brownian motion in general manifolds. See for example the Ref. [43].

This quantization method differs from the others, the canonical and the path integral field quantization, based in the Hamiltonian and the Lagrangian, respectively, in many aspects. The method starts from a classical equation of motion, but not from Hamiltonian or Lagrangian, and consequently can be used to quantize dynamical systems without canonical formalism. Furthermore, it is useful in situations where the others methods lead to difficult problems and can bring us new important results.

For example, a quite important point in a regularization procedure is that it must preserve all the symmetries of the unregularized Lagrangian. Many authors have been stressed that a priori we can not expect that a regularization independent proof of the renormalization of theories in a curved background exists. The presence of the Markov parameter as an extra dimension lead us to a regularization scheme, which preserves all the symmetries of the theory under study. Since the stochastic regularization is not an action regularization, it may be a way to construct such proof. As a starting point of this program, we should calculate the two-point function up to the first order level in the coupling constant $\lambda$ and apply the continuum stochastic regularization. Our results are to be compared with the usual ones in the literature.

The organization of the paper is the following: in section II we discuss the stochastic quantization for the $\left(\lambda \varphi^{4}\right)_{d}$ scalar theory in a $d$-dimensional Euclidean manifold. In section III we use the stochastic quantization and the stochastic regularization to obtain the two-point Schwinger function in the one-loop approximation in the Einstein manifold using the stochastic quantization. In section IV we repeat the method for the case of the Rindler manifold, a non-simple connected manifold in the Euclidean version. Conclusions are given in the section V. In the appendix we sketch the general formalism to construct the maximal analytic extension of the vacuum Schwarzschild solution. In this paper we use $\hbar=c=k_{B}=G=1$. 


\section{Stochastic quantization for the $\left(\lambda \varphi^{4}\right)_{d}$ scalar theory: the Euclidean case}

In this section, we give a brief survey for the case of self-interacting scalar fields, implementing the stochastic quantization and the continuum stochastic regularization theory up to the one-loop level. Let us consider a neutral scalar field with a $\left(\lambda \varphi^{4}\right)$ self-interaction. The Euclidean action that usually describes a free scalar field is

$$
S_{0}[\varphi]=\int d^{d} x\left(\frac{1}{2}(\partial \varphi)^{2}+\frac{1}{2} m_{0}^{2} \varphi^{2}(x)\right),
$$

and the interacting part, defined by the non-Gaussian contribution, is

$$
S_{I}[\varphi]=\int d^{d} x \frac{\lambda}{4 !} \varphi^{4}(x)
$$

The simplest starting point of the stochastic quantization to obtain the Euclidean field theory is a Markovian Langevin equation. Assume a flat Euclidean $d$-dimensional manifold, where we are choosing periodic boundary conditions for a scalar field and also a random noise. In other words, they are defined in a $d$-torus $\Omega \equiv T^{d}$. To implement the stochastic quantization we supplement the scalar field $\varphi(x)$ and the random noise $\eta(x)$ with an extra coordinate $\tau$, the Markov parameter, such that $\varphi(x) \rightarrow \varphi(\tau, x)$ and $\eta(x) \rightarrow \eta(\tau, x)$. Therefore, the fields and the random noise are defined in a domain: $T^{d} \times R^{(+)}$. Let us consider that this dynamical system is out of equilibrium, being described by the following equation of evolution:

$$
\frac{\partial}{\partial \tau} \varphi(\tau, x)=-\left.\frac{\delta S_{0}}{\delta \varphi(x)}\right|_{\varphi(x)=\varphi(\tau, x)}+\eta(\tau, x),
$$

where $\tau$ is a Markov parameter, $\eta(\tau, x)$ is a random noise field and $S_{0}$ is the usual free Euclidean action defined in Eq.(1). For a free scalar field, the Langevin equation reads

$$
\frac{\partial}{\partial \tau} \varphi(\tau, x)=-\left(-\Delta+m_{0}^{2}\right) \varphi(\tau, x)+\eta(\tau, x)
$$

where $\Delta$ is the $d$-dimensional Laplace operator. The Eq.(4) describes a Ornstein-Uhlenbeck process and we are assuming the Einstein relations, that is:

$$
\langle\eta(\tau, x)\rangle_{\eta}=0
$$

and for the two-point correlation function associated with the random noise field

$$
\left\langle\eta(\tau, x) \eta\left(\tau^{\prime}, x^{\prime}\right)\right\rangle_{\eta}=2 \delta\left(\tau-\tau^{\prime}\right) \delta^{d}\left(x-x^{\prime}\right),
$$


where $\langle\ldots\rangle_{\eta}$ means stochastic averages. The above equation defines a delta-correlated random process. In a generic way, the stochastic average for any functional of $\varphi$ given by $F[\varphi]$ is defined by

$$
\langle F[\varphi]\rangle_{\eta}=\frac{\int[d \eta] F[\varphi] \exp \left[-\frac{1}{4} \int d^{d} x \int d \tau \eta^{2}(\tau, x)\right]}{\int[d \eta] \exp \left[-\frac{1}{4} \int d^{d} x \int d \tau \eta^{2}(\tau, x)\right]}
$$

Let us define the retarded Green function for the diffusion problem that we call $G\left(\tau-\tau^{\prime}, x-x^{\prime}\right)$. The retarded Green function satisfies $G\left(\tau-\tau^{\prime}, x-x^{\prime}\right)=0$ if $\tau-\tau^{\prime}<0$ and also

$$
\left[\frac{\partial}{\partial \tau}+\left(-\Delta_{x}+m_{0}^{2}\right)\right] G\left(\tau-\tau^{\prime}, x-x^{\prime}\right)=\delta^{d}\left(x-x^{\prime}\right) \delta\left(\tau-\tau^{\prime}\right) .
$$

Using the retarded Green function and the initial condition $\left.\varphi(\tau, x)\right|_{\tau=0}=0$, the solution for Eq.(4) reads

$$
\varphi(\tau, x)=\int_{0}^{\tau} d \tau^{\prime} \int_{\Omega} d^{d} x^{\prime} G\left(\tau-\tau^{\prime}, x-x^{\prime}\right) \eta\left(\tau^{\prime}, x^{\prime}\right) .
$$

Let us define the Fourier transforms for the field and the noise given by $\varphi(\tau, k)$ and $\eta(\tau, k)$. We have respectively

$$
\varphi(\tau, k)=\frac{1}{(2 \pi)^{\frac{d}{2}}} \int d^{d} x e^{-i k x} \varphi(\tau, x)
$$

and

$$
\eta(\tau, k)=\frac{1}{(2 \pi)^{\frac{d}{2}}} \int d^{d} x e^{-i k x} \eta(\tau, x) .
$$

Substituting Eq.(10) in Eq.(1), the free action for the scalar field in the $(d+1)$-dimensional space writing in terms of the Fourier coefficients reads

$$
\left.S_{0}[\varphi(k)]\right|_{\varphi(k)=\varphi(\tau, k)}=\frac{1}{2} \int d^{d} k \varphi(\tau, k)\left(k^{2}+m_{0}^{2}\right) \varphi(\tau, k) .
$$

Substituting Eq.(10) and Eq.(11) in Eq.(4) we have that each Fourier coefficient satisfies a Langevin equation given by

$$
\frac{\partial}{\partial \tau} \varphi(\tau, k)=-\left(k^{2}+m_{0}^{2}\right) \varphi(\tau, k)+\eta(\tau, k) .
$$

In the Langevin equation the particle is subject to a fluctuating force (representing a stochastic environment), where its average properties are presumed to be known and also the friction force. Note that the "friction coefficient" in the Eq.(13) is given by $\left(k^{2}+m_{0}^{2}\right)$. 
The solution for Eq.(13) reads

$$
\varphi(\tau, k)=\exp \left(-\left(k^{2}+m_{0}^{2}\right) \tau\right) \varphi(0, k)+\int_{0}^{\tau} d \tau^{\prime} \exp \left(-\left(k^{2}+m_{0}^{2}\right)\left(\tau-\tau^{\prime}\right)\right) \eta\left(\tau^{\prime}, k\right) .
$$

Using the Einstein relation, we get that the Fourier coefficients for the random noise satisfies

$$
\langle\eta(\tau, k)\rangle_{\eta}=0
$$

and

$$
\left\langle\eta(\tau, k) \eta\left(\tau^{\prime}, k^{\prime}\right)\right\rangle_{\eta}=2 \delta\left(\tau-\tau^{\prime}\right) \delta^{d}\left(k+k^{\prime}\right) .
$$

It is possible to show that $\left.\left\langle\varphi(\tau, k) \varphi\left(\tau^{\prime}, k^{\prime}\right)\right\rangle_{\eta}\right|_{\tau=\tau^{\prime}} \equiv D\left(k, k^{\prime} ; \tau, \tau^{\prime}\right)$ is given by:

$$
D(k ; \tau, \tau)=(2 \pi)^{d} \delta^{d}\left(k+k^{\prime}\right) \frac{1}{\left(k^{2}+m_{0}^{2}\right)}\left(1-\exp \left(-2 \tau\left(k^{2}+m_{0}^{2}\right)\right)\right) .
$$

where we assume $\tau=\tau^{\prime}$.

Now let us analyze the stochastic quantization for the $\left(\lambda \varphi^{4}\right)_{d}$ self-interaction scalar theory. In this case the Langevin equation reads

$$
\frac{\partial}{\partial \tau} \varphi(\tau, x)=-\left(-\Delta+m_{0}^{2}\right) \varphi(\tau, x)-\frac{\lambda}{3 !} \varphi^{3}(\tau, x)+\eta(\tau, x) .
$$

The two-point correlation function associated with the random field is given by the Einstein relations, while the other connected correlation functions vanish, i.e.,

$$
\left\langle\eta\left(\tau_{1}, x_{1}\right) \eta\left(\tau_{2}, x_{2}\right) \ldots \eta\left(\tau_{2 k-1}, x_{2 k-1}\right)\right\rangle_{\eta}=0
$$

and also

$$
\left\langle\eta\left(\tau_{1}, x_{1}\right) \ldots \eta\left(\tau_{2 k}, x_{2 k}\right)\right\rangle_{\eta}=\sum\left\langle\eta\left(\tau_{1}, x_{1}\right) \eta\left(\tau_{2}, x_{2}\right)\right\rangle_{\eta}\left\langle\eta\left(\tau_{k}, x_{k}\right) \eta\left(\tau_{l}, x_{l}\right)\right\rangle_{\eta} \ldots,
$$

where the sum is to be taken over all the different ways in which the $2 k$ labels can be divided into $k$ parts, i.e., into $k$ pairs. Performing Gaussian averages over the white random noise, it is possible to prove the important formulae

$$
\lim _{\tau \rightarrow \infty}\left\langle\varphi\left(\tau_{1}, x_{1}\right) \varphi\left(\tau_{2}, x_{2}\right) \ldots \varphi\left(\tau_{n}, x_{n}\right)\right\rangle_{\eta}=\frac{\int[d \varphi] \varphi\left(x_{1}\right) \varphi\left(x_{2}\right) \ldots \varphi\left(x_{n}\right) e^{-S(\varphi)}}{\int[d \varphi] e^{-S(\varphi)}}
$$

where $S(\varphi)=S_{0}(\varphi)+S_{I}(\varphi)$ is the $d$-dimensional action. This result leads us to consider the Euclidean path integral measure a stationary distribution of a stochastic process. Note that the solution of the Langevin equation needs a given initial condition. As for example

$$
\left.\varphi(\tau, x)\right|_{\tau=0}=\varphi_{0}(x) .
$$


Let us use the Langevin equation to perturbatively solve the interacting field theory. One way to handle the Eq.(18) is with the method of Green's functions. We defined the retarded Green function for the diffusion problem in the Eq.(8). Let us assume that the coupling constant is a small quantity. Therefore to solve the Langevin equation in the case of a interacting theory we use a perturbative series in $\lambda$. Therefore we can write

$$
\varphi(\tau, x)=\varphi^{(0)}(\tau, x)+\lambda \varphi^{(1)}(\tau, x)+\lambda^{2} \varphi^{(2)}(\tau, x)+\ldots
$$

Substituting the Eq.(23) in the Eq.(18), and if we equate terms of equal power in $\lambda$, the resulting equations are

$$
\begin{gathered}
{\left[\frac{\partial}{\partial \tau}+\left(-\Delta_{x}+m_{0}^{2}\right)\right] \varphi^{(0)}(\tau, x)=\eta(\tau, x)} \\
{\left[\frac{\partial}{\partial \tau}+\left(-\Delta_{x}+m_{0}^{2}\right)\right] \varphi^{(1)}(\tau, x)=-\frac{1}{3 !}\left(\varphi^{(0)}(\tau, x)\right)^{3}}
\end{gathered}
$$

and so on. Using the retarded Green function and assuming that $\left.\varphi^{(q)}(\tau, x)\right|_{\tau=0}=0, \forall q$, the solution to the first equation given by Eq.(24) can be written formally as

$$
\varphi^{(0)}(\tau, x)=\int_{0}^{\tau} d \tau^{\prime} \int_{\Omega} d^{d} x^{\prime} G\left(\tau-\tau^{\prime}, x-x^{\prime}\right) \eta\left(\tau^{\prime}, x^{\prime}\right)
$$

The second equation given by Eq.(25) can also be solved using the above result. We obtain

$$
\begin{aligned}
\varphi^{(1)}(\tau, x)= & -\frac{1}{3 !} \int_{0}^{\tau} d \tau_{1} \int_{\Omega} d^{d} x_{1} G\left(\tau-\tau_{1}, x-x_{1}\right) \\
& \left(\int_{0}^{\tau_{1}} d \tau^{\prime} \int_{\Omega} d^{d} x^{\prime} G\left(\tau_{1}-\tau^{\prime}, x_{1}-x^{\prime}\right) \eta\left(\tau^{\prime}, x^{\prime}\right)\right)^{3} .
\end{aligned}
$$

We have seen that we can generate all the tree diagrams with the noise field contributions. We can also consider the $n$-point correlation function $\left\langle\varphi\left(\tau_{1}, x_{1}\right) \varphi\left(\tau_{2}, x_{2}\right) \ldots \varphi\left(\tau_{n}, x_{n}\right)\right\rangle_{\eta}$. Substituting the above results in the $n$-point correlation function, and taking the random averages over the white noise field using the Wick-decomposition property defined by Eq.(20) we generate the stochastic diagrams. Each of these stochastic diagrams has the form of a Feynman diagram, apart from the fact that we have to take into account that we are joining together two white random noise fields many times. Besides, the rules to obtain the algebraic values of the stochastic diagrams are similar to the usual Feynman rules.

As simple examples let us show how to derive the two-point function in the zeroth order $\left\langle\varphi\left(\tau_{1}, x_{1}\right) \varphi\left(\tau_{2}, x_{2}\right)\right\rangle_{\eta}^{(0)}$, and also the first order correction to the scalar two-point-function given by $\left\langle\varphi\left(\tau_{1}, x_{1}\right) \varphi\left(\tau_{2}, x_{2}\right)\right\rangle_{\eta}^{(1)}$. Using the Eq.(9) and the Einstein relations we have

$$
\left\langle\varphi\left(\tau_{1}, x_{1}\right) \varphi\left(\tau_{2}, x_{2}\right)\right\rangle_{\eta}^{(0)}=2 \int_{0}^{\min \left(\tau_{1}, \tau_{2}\right)} d \tau^{\prime} \int_{\Omega} d^{d} x^{\prime} G\left(\tau_{1}-\tau^{\prime}, x_{1}-x^{\prime}\right) G\left(\tau_{2}-\tau^{\prime}, x_{2}-x^{\prime}\right) .
$$


For the first order correction we get:

$$
\begin{aligned}
& \left\langle\varphi\left(X_{1}\right) \varphi\left(X_{2}\right)\right\rangle_{\eta}^{(1)}= \\
& =-\frac{\lambda}{3 !}\left\langle\int d X_{3} \int d X_{4}\left(G\left(X_{1}-X_{4}\right) G\left(X_{2}-X_{3}\right)+G\left(X_{1}-X_{3}\right) G\left(X_{2}-X_{4}\right)\right)\right. \\
& \left.\eta\left(X_{3}\right)\left(\int d X_{5} G\left(X_{4}-X_{5}\right) \eta\left(X_{5}\right)\right)^{3}\right\rangle_{\eta} .
\end{aligned}
$$

where, for simplicity, we have introduced a compact notation:

$$
\int_{0}^{\tau} d \tau \int_{\Omega} d^{d} x \equiv \int d X
$$

and also $\varphi(\tau, x) \equiv \varphi(X)$ and finally $\eta(\tau, x) \equiv \eta(X)$.

The process can be repeated and therefore the stochastic quantization can be used as an alternative approach to describe scalar quantum fields. Therefore, the two-point function up to the first order level in the coupling constant $\lambda$ is given by

$$
\left\langle\varphi\left(\tau_{1}, x_{1}\right) \varphi\left(\tau_{2}, x_{2}\right)\right\rangle_{\eta}^{(1)}=(a)+(b)+(c),
$$

where $(a)$ is the zero order two-point function and (b) and (c) are given, respectively, by:

$$
\begin{aligned}
& (b)=-\frac{\lambda}{2} \delta^{d}\left(k_{1}+k_{2}\right) \int d^{d} k \int_{0}^{\tau_{1}} d \tau G\left(k_{1} ; \tau_{1}-\tau\right) D(k ; \tau, \tau) D\left(k_{2} ; \tau_{2}, \tau\right), \\
& (c)=-\frac{\lambda}{2} \delta^{d}\left(k_{1}+k_{2}\right) \int d^{d} k \int_{0}^{\tau_{2}} d \tau G\left(k_{2} ; \tau_{2}-\tau\right) D(k ; \tau, \tau) D\left(k_{1} ; \tau_{1}, \tau\right) .
\end{aligned}
$$

These are the contributions in first order. A simple computation shows that we recover the correct equilibrium result at equal asymptotic Markov parameters $\left(\tau_{1}=\tau_{2} \rightarrow \infty\right)$ :

$$
\left.(b)\right|_{\tau_{1}=\tau_{2} \rightarrow \infty}=-\frac{\lambda}{2} \delta^{d}\left(k_{1}+k_{2}\right) \frac{1}{\left(k_{2}^{2}+m_{0}^{2}\right)} \frac{1}{\left(k_{1}^{2}+k_{2}^{2}+2 m_{0}^{2}\right)} \int d^{d} k \frac{1}{\left(k^{2}+m_{0}^{2}\right)} .
$$

Obtaining the Schwinger functions in the asymptotic limit does not guarantee that we gain a finite physical theory. The next step is to implement a suitable regularization scheme. A crucial point to find a satisfactory regularization scheme is to use one that preserves the symmetries of the original model. The presence of the Markov parameter as an extra dimension lead us to a new regularization scheme, the stochastic regularization method, which preserves all the symmetries 
of the theory under study. Therefore, let us implement a continuum regularization procedure [36]. We begin with a regularized Markovian Parisi-Wu Langevin system:

$$
\frac{\partial}{\partial \tau} \varphi(\tau, x)=-\left.\frac{\delta S_{0}}{\delta \varphi(x)}\right|_{\varphi(x)=\varphi(\tau, x)}+\int d^{d} y R_{x y}(\Delta) \eta(\tau, y)
$$

The Einstein relations, given by Eqs. (5) and (6), are maintained. The regulator $R(\Delta)$ that multiplies the noise is a function of the Laplacian:

$$
\Delta_{x y}=\int d^{d} z\left(\partial_{\mu}\right)_{x z}\left(\partial_{\mu}\right)_{z y}
$$

where

$$
\left(\partial_{\mu}\right)_{x y}=\partial_{\mu}^{x} \delta^{d}(x-y) .
$$

We will be working with a heat kernel regulator with the form:

$$
R(\Delta ; \Lambda)=\exp \left(\frac{\Delta}{\Lambda^{2}}\right)
$$

where $\Lambda$ is a parameter introduced to regularize the theory. The basic restrictions on the form of this heat kernel regulator are:

$$
\left.R(\Delta ; \Lambda)\right|_{\Lambda \rightarrow \infty}=1
$$

or

$$
\left.R_{x y}(\Delta ; \Lambda)\right|_{\Lambda \rightarrow \infty}=\delta^{d}(x-y)
$$

which guarantees that the regularized process given by Eq.(35) reduces to the formal process given by Eq.(3) in the formal regulator limit $\Lambda \rightarrow \infty$.

With this modification in the Langevin equation, it is possible to show that all the contributions to the n-point function at all orders in the coupling constant $\lambda$ are finite. For instance, the contribution to the two-point function at the one-loop level given by Eq.(32) is rewritten as:

$$
\left.(b)\right|_{\tau_{1}=\tau_{2} \rightarrow \infty}=-\frac{\lambda}{2} \delta^{d}\left(k_{1}+k_{2}\right) \frac{R_{k_{2}}^{2}}{\left(k_{2}^{2}+m_{0}^{2}\right)} \frac{1}{\left(k_{1}^{2}+k_{2}^{2}+2 m_{0}^{2}\right)} \int d^{d} k \frac{R_{k}^{2}}{\left(k^{2}+m_{0}^{2}\right)},
$$

where $R_{k}$ is the Fourier transform of the regulator, i.e.,

$$
R_{k}(\Lambda)=\left.R(\Delta ; \Lambda)\right|_{\Delta=-k^{2}} .
$$

Now we use this method to discuss the quantization of scalar theories with self-interaction in a curved spacetime without event horizon and in a flat manifold with event horizon. Being more specific, we are interested to investigate the $\lambda \varphi^{4}$ theory in the Einstein and Rindler spacetime, respectively. 


\section{Stochastic quantization for the $\left(\lambda \varphi^{4}\right)_{d}$ scalar theory: the Einstein case}

The aim of this section is to implement the stochastic quantization and the stochastic regularization for the self-interacting $\lambda \varphi^{4}$ theory in the one-loop level in the Einstein spacetime. Let us consider a $M^{4}$ manifold that admit a non-vanishing timelike Killing vector field $X$. If one can always introduce coordinates $t=x^{0}, x^{1}, x^{2}, x^{3}$ locally such that $X=\frac{\partial}{\partial t}$ and the components of the metric tensor are independent of $t, M^{4}$ is stationary. If further the distribution $X^{\perp}$ of 3-planes orthogonal to $X$ is integrable, then $M^{4}$ is static. Each integral curve of the Killing vector vector

field $X=\frac{\partial}{\partial t}$ is a world line of an possible observer. Since $X=\frac{\partial}{\partial t}$ generates isometries, the 3-planes $X^{\perp}$ are invariant under these isometries. For static manifold, it is possible to perform a Wick rotation, i.e., analytically extend the pseudo-Riemannian manifold to the Riemannian domain without problem. Therefore for static spacetime the implementation of the stochastic quantization is straightforward.

In the previous section, we have been working in an Euclidean space $R^{d} \times R^{(+)}$, where $R^{d}$ is the usual Euclidean space and $R^{(+)}$is the Markov sector. Now let us generalize this to a more complicated case, i.e., let us work in a general (and, for the time being, static) Riemannian manifold $M$. In other words, we will consider a classical field theory defined in a $M \times R^{(+)}$ manifold coupled with a heat reservoir. Therefore, in a four-dimensional curved manifold, the Parisi-Wu Langevin equation for scalar fields reads

$$
\frac{\partial}{\partial \tau} \varphi(\tau, x)=-\left.\frac{1}{\sqrt{g}} \frac{\delta S_{0}}{\delta \varphi(x)}\right|_{\varphi(x)=\varphi(\tau, x)}+\eta(\tau, x),
$$

where $g=\operatorname{det} g_{\mu \nu}$ and the classical Euclidean action $S_{0}$ is given by

$$
S_{0}=\int d^{4} x \sqrt{g} \mathcal{L}
$$

In the above equation $\mathcal{L}$ is given by

$$
\mathcal{L}=\frac{1}{2} g_{\mu \nu} \partial_{\mu} \varphi \partial_{\nu} \varphi+\frac{1}{2}\left(m^{2}+\xi R\right) \varphi^{2}
$$

Note that we introduce a coupling between the scalar field and the gravitational field represented by the term $\xi R \varphi^{2}$, where $\xi$ is a numerical factor and $R$ is the Ricci scalar curvature. The random noise field $\eta(\tau, x)$ obeys the following generalized Einstein relations:

$$
\langle\eta(\tau, x)\rangle_{\eta}=0,
$$


and

$$
\left\langle\eta(\tau, x) \eta\left(\tau^{\prime}, x^{\prime}\right)\right\rangle_{\eta}=\frac{2}{\sqrt{g(x)}} \delta^{4}\left(x-x^{\prime}\right) \delta\left(\tau-\tau^{\prime}\right) .
$$

Substituting Eq.(44) and Eq.(45) in the Langevin equation given by Eq.(43), we get:

$$
\frac{\partial}{\partial \tau} \varphi(\tau, x)=-\left(-\Delta+m^{2}+\xi R\right) \varphi(\tau, x)+\eta(\tau, x),
$$

where $\Delta$ is the four-dimensional Laplace-Beltrami operator defined by:

$$
\begin{aligned}
\Delta & =g^{-1 / 2} \partial_{\mu}\left(g^{1 / 2} g_{\mu \nu} \partial_{\nu}\right) \\
& =g_{\mu \nu} \nabla_{\mu} \nabla_{\nu}
\end{aligned}
$$

$\nabla$ denoting the covariant derivative.

To proceed, as in the flat situation, let us introduce the retarded Green function for the diffusion problem $G\left(\tau-\tau^{\prime}, x, x^{\prime}\right)$, which obeys:

$$
\left[\frac{\partial}{\partial \tau}+\left(-\Delta_{x}+m^{2}+\xi R(x)\right)\right] G\left(\tau-\tau^{\prime}, x, x^{\prime}\right)=\frac{1}{\sqrt{g}} \delta^{4}\left(x-x^{\prime}\right) \delta\left(\tau-\tau^{\prime}\right),
$$

if $\tau-\tau^{\prime}>0$, and $G\left(\tau-\tau^{\prime}, x, x^{\prime}\right)=0$ if $\tau-\tau^{\prime}<0$.

Using the retarded Green function and the initial condition $\left.\varphi(\tau, x)\right|_{\tau=0}=0$, a formal solution to Eq.(48) reads

$$
\varphi(\tau, x)=\int_{0}^{\tau} d \tau^{\prime} \int_{\Omega} d^{4} x^{\prime} \sqrt{g\left(x^{\prime}\right)} G\left(\tau-\tau^{\prime}, x-x^{\prime}\right) \eta\left(\tau^{\prime}, x^{\prime}\right) .
$$

We see that, in order to solve Eq.(48) we need to invert the differential operator given by Eq.(49). Depending on the metric $g_{\mu \nu}$ defined on the manifold, this could be a difficult task. In the Euclidean case this is easily solved, since we decompose the fields in Fourier modes (plane waves), which are solution to the Klein-Gordon equation in a flat metric; each Fourier mode, as we have seen, obeys a Langevin equation. So, if the Klein-Gordon equation in a general curved manifold allows us to obtain solutions which can be decomposed in modes separated in a similar manner to the flat case, we may as well decompose our fields in these modes in a way that each of them, again, will obey a Langevin equation. Then, Eq.(48) can be solved in a simple and direct way. Of course, this procedure will help us if we admit manifolds with global Killing vectors, which, unfortunately, is not always the case. So admitting that this is the case, we may write the mode decompositions as:

$$
\varphi(\tau, x)=\int d \tilde{\mu}(k) \varphi_{k}(\tau) u_{k}(x)
$$


and

$$
\eta(\tau, x)=\int d \tilde{\mu}(k) \eta_{k}(\tau) u_{k}(x)
$$

where the measure $\tilde{\mu}(k)$ depends on the metric we are interested in. For instance, in the flat case, we have that in a four dimensional space $d \tilde{\mu}(k)=d^{4} k$ and the modes $u_{k}(x)$ are given by:

$$
u_{k}(x)=\frac{1}{(2 \pi)^{2}} e^{i k x}
$$

So we see that, in the flat case, Eqs. (52) and (53) reduce to the Fourier decomposition given by Eqs.(10) and (11), respectively.

Now let us apply this formalism to the Euclidean Einstein manifold. The general RobertsonWalker line element is given by [44] [45]:

$$
d s^{2}=-d t^{2}+a^{2}(t) \sum_{i, j=1}^{3} h_{i j} d x^{i} d x^{j}
$$

where:

$$
\begin{gathered}
\sum_{i, j=1}^{3} h_{i j} d x^{i} d x^{j}=\left(1-K r^{2}\right)^{-1} d r^{2}+r^{2}\left(d \theta^{2}+\sin ^{2} \phi d \phi^{2}\right) \\
=d \chi^{2}+f^{2}(\chi)\left(d \theta^{2}+\sin ^{2} \phi d \phi^{2}\right),
\end{gathered}
$$

and, for the Einstein manifold we have $(K=1), f(\chi)=r=\sin \chi, 0 \leq \chi \leq 2 \pi$.

The Eq.(56) gives the line element on the spatial sections in the pseudo-Riemannian case which are hyperbolic, flat or closed depending on whether $K=-1,0,1$, respectively. Writing $C(\rho)=a^{2}(t)$, with the conformal time parameter $\rho$ given by

$$
\rho=\int^{t} d t^{\prime} a^{-1}\left(t^{\prime}\right)
$$

the line element can be recast in the form:

$$
d s^{2}=C(\rho)\left(-d \rho^{2}+\sum_{i, j=1}^{3} h_{i j} d x^{i} d x^{j}\right) .
$$

In the simplest case, namely static space-times with $C(\rho)=C=a^{2}=$ constant, the scalar curvature is given by $R=\frac{6 K}{C}$. After the Wick rotation, we are working with the Euclidean Einstein manifold. In this case, we may decompose the modes as:

$$
u_{k}(x)=C^{-1 / 2} \mathbf{X}_{\vec{k}}(\vec{x}) e^{i k_{\rho} \rho}
$$


with $\vec{x}=(r, \theta, \phi)$ or $(\chi, \theta, \phi)$ and $\mathbf{X}_{\vec{k}}$ being a solution of

$$
\triangle^{(3)} \mathbf{X}_{\vec{k}}=-\left(q^{2}-K\right) \mathbf{X}_{\vec{k}} .
$$

In Eq.(60), $\triangle^{(3)}$ is the Laplacian associated with the spatial metric $h_{i j}$ :

$$
\Delta=h^{-1 / 2} \partial_{i}\left(h^{1 / 2} h^{i j} \partial_{j}\right)
$$

Further, the functions $\mathbf{X}_{\vec{k}}$ are normalized such that:

$$
\int d^{3} x h^{\frac{1}{2}} \mathbf{X}_{\vec{k}}(\vec{x}) \mathbf{X}_{k^{\prime}}^{*}(\vec{x})=\delta\left(\vec{k}, \overrightarrow{k^{\prime}}\right)
$$

where $\delta\left(\vec{k}, \overrightarrow{k^{\prime}}\right)$ is the $\delta$-function with respect to the measure $\tilde{\mu}$ :

$$
\int d \tilde{\mu}\left(k^{\prime}\right) f(\vec{k}) \delta\left(\vec{k}, \overrightarrow{k^{\prime}}\right)=f\left(\overrightarrow{k^{\prime}}\right) .
$$

The eigenfunctions $\mathbf{X}_{\vec{k}}$ of the three-dimensional Laplacian are, for $K=1$ :

$$
\mathbf{X}_{\vec{k}}(\vec{x})=\Pi_{q J}^{+}(\chi) Y_{M J}(\theta, \phi)
$$

with $\vec{k}=(q, J, M), M=-J,-J+1, \ldots, J, J=0,1, \ldots, q-1$ and $q=1,2, \ldots$ The $Y_{M J}$ are the usual spherical harmonics. The functions $\Pi_{q J}^{+}$can be defined from [46]:

$$
\Pi_{q J}^{-}(\chi)=\left[\frac{1}{2} \pi q^{2}\left(q^{2}+1\right) \ldots\left(q^{2}+J^{2}\right)\right]^{-1 / 2} \sinh ^{J} \chi\left(\frac{d}{d \cosh \chi}\right)^{J+1} \cos q \chi,
$$

by replacing $q$ by $-i q$ and $\chi$ by $-i \chi$ in the latter.

With these definitions, the measure $\tilde{\mu}(k)$ for the Einstein universe is defined as follows:

$$
\int d \tilde{\mu}(k)=\frac{1}{2 \pi} \int d k_{\rho} \sum_{q, J, M} .
$$

So, inserting the mode decomposition given by Eq.(59) in Eq.(48) we have that each mode coefficient satisfy the Langevin equation given by

$$
\frac{\partial}{\partial \tau} \varphi_{k}(\tau)=-\frac{1}{C}\left(k^{2}+\mu^{2}\right) \varphi_{k}(\tau)+\eta_{k}(\tau)
$$

where $k^{2}=q^{2}+k_{\rho}^{2}$ and $\mu^{2}=C m^{2}+(6 \xi-1) K$ and $K=1$. For simplicity, we redefine $\frac{1}{C}\left(k^{2}+\mu^{2}\right) \rightarrow$ $\left(k^{2}+\mu^{2}\right)$. 
The solution for Eq.(67), with the initial condition $\left.\varphi_{k}(\tau)\right|_{\tau=0}=0$, reads:

$$
\varphi_{k}(\tau)=\int_{0}^{\tau} d \tau^{\prime} G_{k}\left(\tau, \tau^{\prime}\right) \eta\left(\tau^{\prime}, k\right)
$$

where

$$
G_{k}\left(\tau, \tau^{\prime}\right)=\exp \left(-\left(k^{2}+\mu^{2}\right)\left(\tau-\tau^{\prime}\right)\right) \theta\left(\tau-\tau^{\prime}\right)
$$

is the retarded Green function for the diffusion problem.

Using the Einstein relations, we get that the mode coefficients for the random noise satisfies

$$
\left\langle\eta_{k}(\tau)\right\rangle_{\eta}=0
$$

and

$$
\left\langle\eta_{k}(\tau) \eta_{k^{\prime}}\left(\tau^{\prime}\right)\right\rangle_{\eta}=2 \delta\left(\tau-\tau^{\prime}\right) \delta^{4}\left(k, k^{\prime}\right),
$$

where $\delta^{4}\left(k, k^{\prime}\right)=\delta\left(k_{\rho}+k_{\rho}^{\prime}\right) \delta_{q q^{\prime}} \delta_{J J^{\prime}} \delta_{M M^{\prime}}$.

The two-point function $D_{k}\left(\tau, \tau^{\prime}\right)$ can be calculated in a similar way as in the Euclidean flat case. We have

$$
D_{k}\left(\tau, \tau^{\prime}\right)=\frac{1}{(2 \pi)} \delta^{4}\left(k, k^{\prime}\right) \frac{1}{\left(k^{2}+\mu^{2}\right)}\left(e^{-\left(\left(k^{2}+\mu^{2}\right)\left|\tau-\tau^{\prime}\right|\right)}-e^{-\left(\left(k^{2}+\mu^{2}\right)\left(\tau+\tau^{\prime}\right)\right)}\right),
$$

or, in the "coordinate" space:

$$
\begin{aligned}
D\left(\tau, \tau^{\prime} ; x, x^{\prime}\right)= & \int d \tilde{\mu}(k) u_{k}(x) u_{k}^{*}\left(x^{\prime}\right) D_{k}\left(\tau, \tau^{\prime}\right)= \\
& \int d \tilde{\mu}(k) u_{k}(x) u_{k}^{*}\left(x^{\prime}\right) \frac{1}{\left(k^{2}+\mu^{2}\right)}\left(e^{-\left(\left(k^{2}+\mu^{2}\right)\left|\tau-\tau^{\prime}\right|\right)}-e^{-\left(\left(k^{2}+\mu^{2}\right)\left(\tau+\tau^{\prime}\right)\right)}\right),
\end{aligned}
$$

where $\int d \tilde{\mu}(k)$ is given by Eq.(66).

Now, let us apply this method for the case of a self-interacting theory with an interaction action given by:

$$
S_{I}[\varphi]=\int d^{4} x \sqrt{g(x)} \frac{\lambda}{4 !} \varphi^{4}(x) .
$$

In the same way, we can solve the equation using a perturbative series in $\lambda$. The two-point function up to the one loop level is given by:

$$
\left\langle\varphi\left(\tau_{1}, x_{1}\right) \varphi\left(\tau_{2}, x_{2}\right)\right\rangle_{\eta}^{(1)}=(a)+(b)+(c),
$$

where $(a)$ is the zero order two-point function given by Eq.(72) and (b) and (c) are given respectively by

$$
(b)=-\frac{\lambda}{2} \delta^{4}\left(k_{1}, k_{2}\right) \int d \tilde{\beta}(k) \int_{0}^{\tau_{1}} d \tau G_{k_{1}}\left(\tau_{1}-\tau\right) D_{k}(\tau, \tau) D_{k_{2}}\left(\tau_{2}, \tau\right)
$$




$$
(c)=-\frac{\lambda}{2} \delta^{4}\left(k_{1}, k_{2}\right) \int d \tilde{\beta}(k) \int_{0}^{\tau_{2}} d \tau G_{k_{2}}\left(\tau_{2}-\tau\right) D_{k}(\tau, \tau) D_{k_{1}}\left(\tau_{1}, \tau\right)
$$

where $\int d \tilde{\beta}(q) \equiv \frac{1}{2 \pi} \int d k_{\rho} \sum_{q, J, M} \mathbf{X}_{\vec{q}} \mathbf{X}_{\vec{q}}$. These are the contributions in first order. A simple computation shows that we obtain, at equal asymptotic Markov parameters $\left(\tau_{1}=\tau_{2} \rightarrow \infty\right)$ :

$$
\left.(b)\right|_{\tau_{1}=\tau_{2} \rightarrow \infty}=-\frac{\lambda}{2} \delta^{4}\left(k_{1}, k_{2}\right) \frac{1}{\left(k_{2}^{2}+\mu^{2}\right)} \frac{1}{\left(k_{1}^{2}+k_{2}^{2}+2 \mu^{2}\right)} \int d \tilde{\beta}(k) \frac{1}{\left(k^{2}+\mu^{2}\right)} .
$$

Now we define the quantity $I$ as:

$$
\begin{aligned}
I= & \int d \tilde{\beta}(q) \frac{1}{\left(k^{2}+\mu^{2}\right)} \\
& =\frac{1}{2 \pi} \int d k_{\rho} \sum_{q, J, M} \mathbf{X}_{\vec{q}} \mathbf{X}_{\vec{q}} \frac{1}{\left(k^{2}+\mu^{2}\right)} \\
& =\int d k_{\rho} \sum_{q} \mathbf{X}_{\vec{q}} \mathbf{X}_{\vec{q}} \frac{q^{2}}{\left(q^{2}+b^{2}\right)}
\end{aligned}
$$

where $b^{2}=k_{\rho}^{2}+\mu^{2}$. It is easy to show that the series in this equation is divergent. So, we need a procedure to regularize it and obtain a finite quantity for the two-point function. As we will see now, this can be done within the covariant stochastic regularization [36].

The generalization of Eq.(35) to general four-dimensional spacetimes is

$$
\frac{\partial}{\partial \tau} \varphi(\tau, x)=-\left.\frac{1}{\sqrt{g}} \frac{\delta S_{0}}{\delta \varphi(x)}\right|_{\varphi(x)=\varphi(\tau, x)}+\int d^{4} y \sqrt{g} R_{x y}(\Delta) \eta(\tau, y),
$$

where, for covariant reasons, the regulator is now a function of the covariant Laplacian:

$$
\Delta_{x y}=\int d^{4} z\left(\nabla_{\mu}\right)_{x z}\left(\nabla_{\mu}\right)_{z y}
$$

with

$$
\left(\nabla_{\mu}\right)_{x y}=\nabla_{\mu}^{x} \delta^{4}(x-y)
$$

Using the mode decomposition given by Eq.(59), the Eq.(80) reduces to

$$
\frac{\partial}{\partial \tau} \varphi_{k}(\tau)=-\frac{1}{C}\left(k^{2}+\mu^{2}\right) \varphi_{k}(\tau)+\eta_{k}(\tau) R_{k}
$$

where $R_{k}=\left.R_{x y}(\Delta)\right|_{\Delta=-k^{2}+K}$ and

$$
R_{x y}(\Delta)=\int d \tilde{\mu}(k) u_{k}(x) u_{k}^{*}(y) R_{k} .
$$


Then, the solution for the regularized Langevin equation follows:

$$
\varphi_{k}(\tau)=\int_{0}^{\tau} d \tau^{\prime} G_{k}\left(\tau, \tau^{\prime}\right) \eta\left(\tau^{\prime}, k\right)
$$

where $G_{k}\left(\tau, \tau^{\prime}\right)$ is given by Eq.(69). Then, it is easy to show that the zero order two-point function and the regularized contribution $(b)_{\Lambda}$ at the first order at the coupling constant are given by, respectively:

$$
D_{k}\left(\tau, \tau^{\prime}\right)=\frac{1}{(2 \pi)} \delta^{4}\left(k, k^{\prime}\right) \frac{R_{k}^{2}}{\left(k^{2}+\mu^{2}\right)}\left(e^{-\left(\left(k^{2}+\mu^{2}\right)\left|\tau-\tau^{\prime}\right|\right)}-e^{-\left(\left(k^{2}+\mu^{2}\right)\left(\tau+\tau^{\prime}\right)\right)}\right),
$$

and

$$
\left.(b)_{\Lambda}\right|_{\tau_{1}=\tau_{2} \rightarrow \infty}=-\frac{\lambda}{2} \delta^{4}\left(k_{1}, k_{2}\right) \frac{R_{k_{2}}^{2}}{\left(k_{2}^{2}+\mu^{2}\right)} \frac{1}{\left(k_{1}^{2}+k_{2}^{2}+2 \mu^{2}\right)} \int d \tilde{\beta}(k) \frac{R_{k}^{2}}{\left(k^{2}+\mu^{2}\right)} .
$$

Let us isolate the part where we had problems. We have:

$$
\begin{aligned}
I_{\Lambda}= & \int d \tilde{\beta}(k) \frac{R_{k}^{2}}{\left(k^{2}+\mu^{2}\right)} \\
& =\frac{1}{2 \pi} \int d k_{\rho} \sum_{q, J, M} \mathbf{X}_{\vec{q}} \mathbf{X}_{\vec{q}} \frac{R_{k}^{2}}{\left(k^{2}+\mu^{2}\right)} .
\end{aligned}
$$

After some tedious calculations [47] [48] [49], we arrive at:

$$
I_{\Lambda}=\frac{1}{4} e^{\frac{2 K}{C \Lambda^{2}}+\frac{2}{C \Lambda^{2}} \mu^{2}} \int_{\mu}^{\infty} d x\left(x^{2}-\mu^{2}\right)^{1 / 2} \operatorname{erfc}(\sqrt{\alpha} x)
$$

where $\operatorname{erf} c(x)$ is the complementary error function. At the expense of considerable labor, it is possible to extend our results to the four-point function.

A quite important point is that this regularization procedure preserves all the symmetries of the unregularized Lagrangian, since it is not an action regularization. The next step would be to isolate the parts that go to infinity in the limit $\Lambda \rightarrow \infty$ and remove them with a suitable redefinition of the constants of the theory, i.e., carry out the renormalization program. A natural question now would be if we can actually renormalize all the $n$-point functions at all orders at the coupling constant $\lambda$. Birrel [50] has given arguments that a priori we cannot expect that a regularization independent proof of the renormalizability of the $\lambda \varphi^{4}$ theory in a curved background exists. One attempt of general proof of renormalizability of $\lambda \varphi^{4}$ theory defined in a spacetime which can be analytically continued to Euclidean situation was given by Bunch [51]. Using the Epstein-Glaser method, Brunetti and Fredenhagen [52] presented a perturbative construction of this theory on a smooth globally hyperbolic curved spacetime. 
Our derivation shows that the stochastic regularization may be an attempt in a direction of such regularization independent proof, even though we are still restricted to the same situation studied by Bunch. Indeed, we know that, in this case of the Einstein universe, the free two-point function presents itself as a sum of Minkowski space positive frequency two-point functions, so it is natural to expect that, as it is in the Minkowski case, a regularization independent proof of the renormalizability of such interacting theory does exist, at least for the Einstein universe.

\section{Stochastic quantization for the $\left(\lambda \varphi^{4}\right)_{d}$ scalar theory: the Rindler case}

Now let us consider the stochastic quantization in a spacetime with an event horizon. The Schwarzschild black hole is the most familiar solution to the vacuum Einstein equations, which has an event horizon. At the origin, there is a curvature singularity and at $R=2 M$ we have a singularity due to the bad behavior of this particular coordinate system. Although it is not possible to extend the spacetime across the singularity, it is possible to analytically continue the manifold from $r>2 M$ to the region $r<2 M,(r \neq 0)$. The maximal extension of the manifold described by the Schwarzschild line element with $2 M<r<\infty$ is the usual Kruskal extension [53]. The Kruskal spacetime defines two outer asymptotically flat regions and also two regions inside the event horizon, bounded by the past and future singularities (see Appendix A). Since we know that, close to the horizon, the Schwarzschild coordinates $t$ and $r$ behaves as Rindler's spacetime coordinates, we shall investigate the stochastic quantization in the Rindler metric. The background material relevant for us can be found in the text book [54] and also in the review articles [55] [56] [57] [58].

Let us consider a d-dimensional flat Minkowski spacetime where we are using the usual cartesian coordinates $y^{\mu}=\left(y^{0}, y^{1}, \ldots, y^{d-1}\right)$. It is possible to find a curvilinear coordinate system $x^{\mu}=$ $\left(t, x^{1}, \ldots, x^{d-2}, z\right)$, called the Rindler's coordinate system and can be shown that this coordinate system is one naturaly adapted to an observer with constant proper acceleration. It is important to stress that this coordinate system with the respective coordinate transformation cover only the region $y^{d-1}>\left|y^{0}\right|$. Since this coordinate system does not cover the whole Minkowski spacetime, we can define three coordinate transformations with the respective coordinate systems defined in different regions of the Minkowski spacetime. These regions are known in the literature as Rindler's L, Milne F, and finally Milne P. The four coordinate transformations and the coordinate systems together cover all the Minkowski spacetime. The coordinate system that cover the region inside the light cone is the $d$-dimensional Milne spacetime.

The implementation of the canonical quantization in Rindler's spacetime is very simple, since if 
the spacetime has a stationary geometry, there is a time-like killing vector field $K$ that generates one-parametric Lie group of isometries and the orthogonal modes which satisfies $\mathcal{L}_{K} u_{n}(x)=$ $-i \omega u_{n}$, where $\mathcal{L}_{K}$ is the Lie derivative with respect to $K$ and the $u_{n}(x)$ are the positive frequency modes. In this situation, there is a natural way to define positive and negative frequency modes. Note that the Rindler's line element is $t$ independent, and consequently there is a straightforward way to define positive and negative frequency modes in order to impose the canonical quantization in Rindler's spacetime.

It has long be recognized that the Rindler's vacuum $|0, R\rangle$ that appear in the Fulling canonical quantization of a scalar field in the Rindler's spacetime is not unitarily equivalent to the Minkowski vacuum $\mid 0, M>$. Let us define the following generating functionals, $Z(h)$ and $Z_{R}(h)$, i.e.,

$$
Z(h)=\frac{\langle 0, M, \text { out }| 0, M, \text { in }\rangle_{h}}{\langle 0, M, \text { out }| 0, M, \text { in }\rangle_{h=0}}
$$

and

$$
Z_{R}(h)=\frac{\langle 0, R, \text { out }| 0, R, \text { in }\rangle_{h}}{\langle 0, R, \text { out }| 0, R, \text { in }\rangle_{h=0}}
$$

where $\mid 0, M$, in $\rangle, \mid 0, M$, out $\rangle, \mid 0, R$, in $\rangle$ and finally $|0, R, o u t\rangle$ are the IN and OUT vacuum states for the theory with the Minkowski and Rindler Hamiltonian density respectively.

Starting from $Z(h)$ it is possible after analytic continuation and imposing periodicity in the Euclidean time, to define $Z(\beta ; h)$, i.e., the finite temperature Schwinger functional. It is clear that $\lim _{\beta \rightarrow \infty} Z(\beta ; h)=Z(\infty ; h) \equiv Z(h)$, where $Z(\infty ; h)$ is the zero temperature Euclidean functional which generates the Schwinger functions in the whole Euclidean space. At the same way it is possible to define $Z_{R}(\beta ; h)$, i.e. the finite temperature Euclidean Rindler functional. Again we have $\lim _{\beta \rightarrow \infty} Z_{R}(\beta ; h)=Z_{R}(\infty ; h) \equiv Z(h)$, where $Z_{R}(\infty ; h)$ is the zero temperature Euclidean functional which generates the Schwinger functions in the analytic extended Rindler manifold.

From the above definitions, let us define the following Schwinger functions. We are following the discussion of Christensen and Duff [59]:

i) $G_{\beta}\left(x, x^{\prime}\right)$ two-point function, i.e. the Schwinger function obtained from the finite temperature Schwinger functional.

ii) $G_{\infty}\left(x, x^{\prime}\right)$ two-point function, i.e. the Schwinger function obtained from the zero temperature Schwinger functional.

Now, let us analyse the two-point function that we are able to construct in the Euclidean manifold. Remember that the Rindler Euclidean metric possesses a coordinate singularity at the origin where $z=0$. The point $z=0$ is a conical point of the Euclidean manifold but is a regular point if the Euclidean time is periodic.

i) $G^{(1)}\left(x, x^{\prime}\right)$ two-point function which is periodic in the Euclidean Rindler's time. This is the usual scalar two-point Schwinger function. It is a function of the geodesic distance beween two 
points in the manifold. Since all path around the origin are topologically equivalent, therefore $G^{(1)}\left(x, x^{\prime}\right)$ should be periodic in Euclidean Rindler's time. Therefore these Green's functions can be generated by functional derivatives in the $Z(\beta ; h)$, i.e., the finite temperature Schwinger functional.

ii) $G^{(0)}\left(x, x^{\prime}\right)$ two-point function. This two-point function appears in the situation where the Euclidean space has a hole in the origin. In the case where the two points are on the circular world-line it is possible to relate the two two-point functions $G^{(1)}\left(x, x^{\prime}\right)$ and $G^{(0)}\left(x, x^{\prime}\right)$. This can be done using a simple trigonometric identity

$$
G^{(1)}\left(\tau, \tau^{\prime}\right)=\sum_{n=-\infty}^{\infty} G^{0}\left(\tau, \tau^{\prime}+\frac{2 \pi n}{a}\right) .
$$

Since the finite temperature Schwinger function must be periodic in the Euclidean time with periodicity $\beta$, making the identification $\beta=\frac{2 \pi}{a}$ it is clear that $G^{(1)}\left(x, x^{\prime}\right)=G_{\beta}\left(x, x^{\prime}\right)$. Also we have $G^{(0)}\left(x, x^{\prime}\right)=G_{\infty}\left(x, x^{\prime}\right)$. Therefore these Green's functions can be generated by functional derivatives in the $Z(\infty ; h)$ which is the zero temperature Euclidean functional which generates the Schwinger functions in the whole Euclidean space.

Now, after a Wick rotation, we should apply the stochastic quantization for the four-dimensional Rindler space, which becomes a multiple connected manifold, with the Euclidean metric:

$$
d s^{2}=z^{2} d t^{2}+d x_{1}^{2}+d x_{2}^{2}+d z^{2}
$$

In the Euclidean Rindler space, the Langevin equation for the field $\varphi(x)$ reads:

$$
\frac{\partial}{\partial \tau} \varphi(\tau, x)=-\left.\sqrt{g} \frac{\delta S_{0}}{\delta \varphi(x)}\right|_{\varphi(x)=\varphi(\tau, x)}+\eta(\tau, x),
$$

where $S_{0}$ is the Euclidean action for the scalar free fields. Notice that this expression is quite different from the Langevin equation for the Einstein metric, Eq.(43), since we have inverse power of the determinant of the metric multiplied by $\frac{\delta S_{0}}{\delta \varphi(x)}$. So, with the Rindler metric given above, we have:

$$
\frac{\partial}{\partial \tau} \varphi(\tau, x)=-\left(-\Delta+z^{2} m^{2}\right) \varphi(\tau, x)+\eta(\tau, x)
$$

where the operator $\Delta$ is defined by:

$$
\Delta=\partial_{t}^{2}+z^{2}\left(\partial_{x_{1}}^{2}+\partial_{x_{2}}^{2}+\partial_{z}^{2}\right)+z \partial_{z}
$$

To proceed with the implementation of the stochastic quantization, we have to use the generalized Einstein relations, given by Eq.(46) and Eq.(47). 
From the modes presented by references [60] [61], we can obtain the following Euclidean modes:

$$
u_{k \nu}(\vec{x}, z)=\frac{1}{2 \pi^{2}}(\nu \sinh (\pi \nu))^{1 / 2} e^{i \vec{k} \cdot \vec{x}} K_{i \nu}(\mu z)
$$

where $\vec{k} \cdot \vec{x}=k_{0} t+k_{1} x_{1}+k_{2} x_{2}, \mu=\sqrt{\vec{k}^{2}+m^{2}}$ and $K_{\mu}(x)$ is the Macdonald function. This are normalized such as:

$$
\frac{1}{\pi^{2}} \int \frac{d z}{z} K_{i \nu}(\mu z) K_{i \nu^{\prime}}(\mu z)=\frac{\delta\left(\nu, \nu^{\prime}\right)}{\left(\nu+\nu^{\prime}\right)\left(\sinh (\pi \nu) \sinh \left(\pi \nu^{\prime}\right)\right)^{1 / 2}} .
$$

Now, we use the general mode decomposition, given by Eq.(52) and Eq.(53), with the following measure:

$$
\int d \tilde{\mu}(k)=\int_{0}^{\infty} d \nu \int d \vec{k}=\int_{0}^{\infty} d \nu \int d k_{1} \int d k_{2} \int \frac{d k_{0}}{2 \pi}
$$

Remembering Eq.(97) for the modes, we will have again that each mode coefficient obeys a Langevin equation of the form:

$$
\frac{\partial}{\partial \tau} \varphi_{k \nu}(\tau)=-\left(k_{0}^{2}+\nu^{2}\right) \varphi_{k \nu}(\tau)+\eta_{k \nu}(\tau)
$$

The solution for Eq.(100), with the initial condition $\left.\varphi_{k \nu}(\tau)\right|_{\tau=0}=0$, reads:

$$
\varphi_{k \nu}(\tau)=\int_{0}^{\tau} d \tau^{\prime} G_{k \nu}\left(\tau, \tau^{\prime}\right) \eta\left(\tau^{\prime}, k\right)
$$

where

$$
G_{k \nu}\left(\tau, \tau^{\prime}\right)=\exp \left(-\left(k_{0}^{2}+\nu^{2}\right)\left(\tau-\tau^{\prime}\right)\right) \theta\left(\tau-\tau^{\prime}\right)
$$

is the retarded Green function for the diffusion problem. Using the Einstein relations, we get that the mode coefficients for the random noise satisfies

$$
\left\langle\eta_{k}(\tau)\right\rangle_{\eta}=0
$$

and

$$
\left\langle\eta_{k}(\tau) \eta_{k^{\prime}}\left(\tau^{\prime}\right)\right\rangle_{\eta}=2 \delta\left(\tau-\tau^{\prime}\right) \delta\left(k, k^{\prime}\right) \delta\left(\nu, \nu^{\prime}\right)
$$

where $\delta\left(k, k^{\prime}\right)=\delta\left(k_{0}+k_{0}^{\prime}\right) \delta\left(\vec{k}+\overrightarrow{k^{\prime}}\right)$.

The two-point function $D_{k}^{0}\left(\tau, \tau^{\prime}\right)$ can be calculated in a similar way as in the Einstein case. We have

$$
D_{k \nu}^{0}\left(\tau, \tau^{\prime}\right)=\delta\left(k, k^{\prime}\right) \delta\left(\nu, \nu^{\prime}\right) \frac{1}{\left(k_{0}^{2}+\nu^{2}\right)}\left(e^{-\left(\left(k_{0}^{2}+\nu^{2}\right)\left|\tau-\tau^{\prime}\right|\right)}-e^{-\left(\left(k_{0}^{2}+\nu^{2}\right)\left(\tau+\tau^{\prime}\right)\right)}\right)
$$


or, in the "coordinate" space:

$$
\begin{aligned}
D^{0}\left(x, x^{\prime} ; \tau, \tau^{\prime}\right)= & \frac{1}{4 \pi^{4}} \int_{0}^{\infty} d \nu \int d k \nu \sinh (\pi \nu) K_{i \nu}(\mu z) K_{i \nu}\left(\mu z^{\prime}\right) \\
& \frac{e^{i \vec{k} .\left(\vec{x}-\overrightarrow{x^{\prime}}\right)}}{k_{0}^{2}+\nu^{2}}\left(e^{-\left(\left(k_{0}^{2}+\nu^{2}\right)\left|\tau-\tau^{\prime}\right|\right)}-e^{-\left(\left(k_{0}^{2}+\nu^{2}\right)\left(\tau+\tau^{\prime}\right)\right)}\right) .
\end{aligned}
$$

In the limit $\tau=\tau^{\prime} \rightarrow \infty$, and proceeding with similar steps as in reference [60], one may prove that we have obtained the usual result for the free two-point function, i.e., the Schwinger two-point function for a (straight-line) geodesic distance between the points $x$ e $x^{\prime}$. As is well known [59], this function is determined uniquely by the requirement that, in the absence of any holes in space, one identifies the "angle" $t$ (which is the Euclidean "time") with the angle $t+2 \pi n$ ( $n=$ integer) because all paths around the origin are topologically equivalent. Thus, as expected, $D^{0}$ should be periodic in $t$ with period $2 \pi$. But we know that, when we do the analytical continuation $t \rightarrow i t$ in the Rindler metric, the event horizon, which in Minkwoski space is represented as an impenetrable barrier to the accelerating observer, translates in Euclidean language into the statement that closed paths around the origin cannot be continuously shrunk to a point. An inertial observer sees a space with the usual topology, $R^{4}$, but the topology seen by the accelerating observer is different, i.e., his space has a hole in it. Then, paths winding around the origin cannot all be deformed into each other; they fall into topologically distinct classes labelled by the winding number $n$. Now, we do not identify $t$ with $t+2 \pi n$. This two-point function, which we shall call $D^{1}$, exhibits an infinite periodicity. To obtain it, we should work with the following modes [62]:

$$
u_{k \nu}(\vec{x}, z)=\frac{1}{2 \pi^{2}}(\nu \sinh (\pi \nu))^{1 / 2} e^{i\left(k_{1} x_{1}+k_{2} x_{2}\right)-k_{0}|t|} K_{i \nu}(\mu z),
$$

With this prescription, we obtain the same results as found in literature [63]:

$$
\begin{aligned}
D^{0}\left(x, x^{\prime} ; \tau, \tau^{\prime}\right)= & \sum_{n=-\infty}^{n=+\infty} D^{1}\left(x, x^{\prime} ; \tau, \tau^{\prime}\right) \\
& =\frac{1}{4 \pi^{4}} \sum_{n=-\infty}^{n=+\infty} \int_{0}^{\infty} d \nu \int d k \nu \sinh (\pi \nu) K_{i \nu}(\mu z) K_{i \nu}\left(\mu z^{\prime}\right) \\
& \frac{e^{i\left(k_{1} x_{1}+k_{2} x_{2}\right)-k_{0}|t+2 \pi n|}}{k_{0}^{2}+\nu^{2}}\left(e^{-\left(\left(k_{0}^{2}+\nu^{2}\right)\left|\tau-\tau^{\prime}\right|\right)}-e^{-\left(\left(k_{0}^{2}+\nu^{2}\right)\left(\tau+\tau^{\prime}\right)\right)}\right),
\end{aligned}
$$

where the geodesic distance between the points $x$ and $x^{\prime}$ is an arc length in the function $D^{1}$. It is interesting to note that, using the modes given by Eq.(107) (see reference [62]), and then summing 
up all configurations with a winding number $n$ we reach the same result as using the modes given by Eq.(97) (see reference [60]).

Let us repeat the method for the case of self-interacting theory with the interaction action given by:

$$
S_{I}[\varphi]=\int d^{4} x \sqrt{g(x)} \frac{\lambda}{4 !} \varphi^{4}(x) .
$$

In this case, we have similar equations to the Einstein case. Eq.(32) reads, in the Rindler space:

$$
(b)=-\frac{\lambda}{2} \delta\left(k_{1}, k_{2}\right) \delta\left(\nu_{1}, \nu_{2}\right) \int d \tilde{\beta}(k) \int_{0}^{\tau_{1}} d \tau G_{k_{1} \nu_{1}}\left(\tau_{1}-\tau\right) D_{k \nu}^{0}(\tau, \tau) D_{k_{2} \nu_{2}}^{0}\left(\tau_{2}, \tau\right)
$$

which, in the limit $\tau=\tau^{\prime} \rightarrow \infty$, becomes:

$$
\left.(b)\right|_{\tau_{1}=\tau_{2} \rightarrow \infty}=-\frac{\lambda}{2} \delta\left(k_{1}, k_{2}\right) \delta\left(\nu_{1}, \nu_{2}\right) \frac{1}{\left(k_{2}^{2}+\nu_{2}^{2}\right)} \frac{1}{\left(k_{1}^{2}+k_{2}^{2}+\nu_{1}^{2}+\nu_{2}^{2}\right)} \int d \tilde{\beta}(k) \frac{1}{\left(k^{2}+\nu^{2}\right)}
$$

with similar divergences and $\int d \tilde{\beta} \equiv \int d \nu \int d k \nu \sinh (\pi \nu) K_{i \nu}(\mu z) K_{i \nu}(\mu z)$. Now, we may apply the continuum regularization as before [36], with a regulator that is a function of the following operator:

$$
\Delta^{\prime}=z^{2}\left(\partial_{x_{1}}^{2}+\partial_{x_{2}}^{2}+\partial_{z}^{2}\right)+z \partial_{z}-z^{2} m^{2}
$$

The regularized Langevin equation reads:

$$
\frac{\partial}{\partial \tau} \varphi_{k \nu}(\tau)=-\left(k_{0}^{2}+\nu^{2}\right) \varphi_{k \nu}(\tau)+R_{k \nu} \eta_{k \nu}(\tau)
$$

with the solution:

$$
\varphi_{k \nu}(\tau)=\int_{0}^{\tau} d \tau^{\prime} G_{k \nu}\left(\tau, \tau^{\prime}\right) R_{k \nu} \eta\left(\tau^{\prime}, k\right) .
$$

where $R_{k \nu}(\Lambda)=\left.R\left(\Delta^{\prime} ; \Lambda\right)\right|_{\Delta^{\prime}=\nu^{2}}$. Then, it is easy to show that the zero order two-point function and the regularized contribution $(b)_{\Lambda}$ at the one loop level are given by, respectively:

$$
D_{k \nu}^{\Lambda}\left(\tau, \tau^{\prime}\right)=\delta\left(k, k^{\prime}\right) \delta\left(\nu, \nu^{\prime}\right) \frac{R_{k \nu}^{2}}{\left(k_{0}^{2}+\nu^{2}\right)}\left(e^{-\left(\left(k_{0}^{2}+\nu^{2}\right)\left|\tau-\tau^{\prime}\right|\right)}-e^{-\left(\left(k_{0}^{2}+\nu^{2}\right)\left(\tau+\tau^{\prime}\right)\right)}\right)
$$

and

$$
\left.(b)\right|_{\tau_{1}=\tau_{2} \rightarrow \infty}=-\frac{\lambda}{2} \delta\left(k_{1}, k_{2}\right) \delta\left(\nu_{1}, \nu_{2}\right) \frac{R_{k_{2} \nu_{2}}^{2}}{\left(k_{2}^{2}+\nu_{2}^{2}\right)} \frac{1}{\left(k_{1}^{2}+k_{2}^{2}+\nu_{1}^{2}+\nu_{2}^{2}\right)} \int d \tilde{\beta}(k) \frac{R_{k \nu}^{2}}{\left(k^{2}+\nu^{2}\right)} .
$$


So, the regularized part of the contribution above reads:

$$
I=\frac{1}{4 \pi^{4}} \int_{0}^{\infty} d \nu \int_{0}^{\infty} d \vec{k} \int \frac{d k_{0}}{2 \pi} \nu \sinh (\pi \nu) K_{i \nu}(\mu z) K_{i \nu}(\mu z) \frac{e^{\frac{-2 \nu^{2}}{\Lambda^{2}}}}{k_{0}^{2}+\nu^{2}}
$$

With some tedious manipulations [47] [48] [49], we arrive at:

$$
I=\left(\frac{\pi}{2}\right)^{1 / 2} \frac{\Lambda m}{16} e^{\frac{\pi^{2} \Lambda^{2}}{8}} f(\Lambda, z)
$$

where the function $f(\Lambda, z)$ is given by

$$
f=\int_{-\infty}^{+\infty} \frac{d \alpha}{\gamma} K_{1}(m \gamma) e^{-\frac{\Lambda^{2} \alpha^{2}}{8}}\left(e^{\frac{i \pi \alpha \Lambda^{2}}{4}} \operatorname{erfc}\left(-\frac{\Lambda}{2 \sqrt{2}}(i \alpha+\pi)\right)-e^{-\frac{i \pi \alpha \Lambda^{2}}{4}} \operatorname{erfc}\left(-\frac{\Lambda}{2 \sqrt{2}}(i \alpha-\pi)\right)\right)
$$

where $\gamma^{2}=2 z^{2}(1+\cosh \alpha)$ and $\operatorname{erfc}(x)$ is the complementary error function, which satisfies the identity:

$$
\operatorname{erfc}(-x)=2-\operatorname{erfc}(x)
$$

It is not possible to present the solution of the integral in Eq.(119) in terms of known functions. In spite of this inconvenience, it is easy to see that this function $f(\Lambda, z)$ has strong convergence, proving that we indeed regularize the contribution for the two-point function at the one-loop level. Similar calculations can be carried out for the four-point function. From the discussions it should be clear that is a simple matter to derive the Schwinger functions of the theory at least in the one-loop level.

There are two rather subtle points that we have to investigate. The first one is related to the behavior of the random noise near event horizon, and the second one is the validity of the program beyond the Euclidean signature.

\section{Conclusions and perspectives}

The stochastic quantization method was used to study self-interacting fields in manifolds which can be analytically continued to the Euclidean situation, i.e., static Riemannian manifolds, namely, the Einstein manifold and the Rindler manifold. First, we have solved a Langevin equation for the mode coefficients of the field, then we exhibit the two-point function at the one-loop level. It was shown that it diverges and we have used a covariant stochastic regularization to regularize it. It was shown that, indeed, the two-point function is regularized. 
A natural question that arises, when we work in Rindler space, is that what happens to the noise field correlation function, given by Eq.(47), near an horizon. From this equation, we see that, whenever we have $g=\operatorname{det} g_{\mu \nu}=0$, this correlation function diverges, and, therefore, all n-point correlation functions $\left\langle\varphi\left(\tau_{1}, x_{1}\right) \varphi\left(\tau_{2}, x_{2}\right) \ldots \varphi\left(\tau_{n}, x_{n}\right)\right\rangle_{\eta}$ will have meaningless values, in virtue of the solution of the Langevin equation. We may implement a brick wall-like model [64] [65] in order to account for these effects; in other words, we may impose a boundary condition on solutions of the Langevin equation at a point near the horizon. On the other hand, in the limit $g=\operatorname{det} g_{\mu \nu} \rightarrow \infty$, all the correlation functions vanish. We would like to point out that another and powerful way to understand the behavior of the correlation functions in the limit $g=\operatorname{det} g_{\mu \nu}=0$ may be the stochastic quantization of the two-dimensional Schwinger model in a curved background. This can also be a way to study the most pertinent form of the Langevin equation to be used in curved spacetimes. This subject and also the link between our results and the program to implement the perturbative renormalization in domains where the translational invariance is broken [66] [67] [68] [69] is under investigation by the authors.

We discussed also that the presence of the Markov parameter as an extra dimension lead us to a regularization scheme, which preserves all the symmetries of the theory under study. This is a quite important point in a regularization procedure. It must preserve all the symmetries of the unregularized Lagrangian. Although many authors have stressed that we can not expect that a regularization independent proof of the renormalization of theories in a curved background exists, since the stochastic regularization is not an action regularization, may be a way to construct such proof. We are aware of the fact that the stochastic quantization program can be implemented without problems (modulo event horizon problems, etc), if it is possible to perform the Wick rotation, obtaining a real Euclidean action.

The picture that emerges from the discussions of the paper is that the implementation of the stochastic quantization in curved background is related to the following fact. For static manifold, it is possible to perform a Wick rotation, i.e., analytically extend the pseudo-Riemannian manifold to the Riemannian domain without problem. Nevertheless, for non-static curved manifolds we have to extend the formalism beyond the Euclidean signature, i.e., to formulate the stochastic quantization in pseudo-Riemannian manifold, not in the Riemannian space (as in the original Euclidean space) as was originally formulated. See for example the Refs. [11] [12]. In the Ref. [11], the authors proposed a modification of the original Parisi-Wu scheme, introducing a complex drift term in the Langevin equation, to implement the stochastic quantization in Minkowski spacetime. Gozzi [12] studied the spectrum of the non-self-adjoint Fokker-Planck Hamiltian to justify this program. See also the Refs. [70] [71]. Of course, this situation is a special case of ordinary Euclidean formulation for systems with complex actions.

The main difference between the original Euclidean and the implementation of the stochastic quantization in Minkowski spacetime is the fact that in the usual case a real Euclidean action the 
approach to the equilibrium state is a stationary solution of the Focker-Planck equation. In the Minkowski formulation, the Hamiltonian is non-Hermitian and the eigenvalues of such Hamiltonian are in general complex value. The real part of such eigenvalues are important to the asymptotic behavior at large Markov time. The approach to the equilibrium is achieved only if we can show its positive semi-definiteness. The fundamental question is: what happens if the Langevin equation describes diffusion around complex action? Some authors claim that it is possible to obtain meaningful results out of Langevin equation diffusion processes around complex action. See for example the seminal paper of Parisi [72]. Klauder et al. [73] investigated the complex Lagevin equation, where some numerical simulations in one-dimensional systems was presented. See also the Refs. [74] [75]. Finally we would like to mention the approach developed by Okamoto et al. [76], where the role of the kernel in the complex Langevin equation was studied.

We would like to remark that there are many examples where Euclidean action is complex. The stochastic quantization in Minkowski spacetime, as we discussed; system with chemical potential as for example $Q C D$ with non-vanishing chemical potential at finite temperature; for $S U(N)$ theories with $N>2$, the fermion determinant becomes complex and also the effective action. Complex terms can also appears in the Langevin equation for fermions, but a suitable kernel can circumvent this problem. Another quite instructive case that deserves our attention is the stochastic quantization of topological field theory. One of the peculiar feature within this kind of theory is the appearance of a factor $i$ in front of the topological action in Euclidean space. In a topological theory, the path integral measure weighing remains to be $e^{i S}$, even after the Wick rotation. An attempt to use a Markovian Langevin equation with a white noise to quantize the theory, fails since the Langevin equation will not tend to any equilibrium at large Markov parameter. In the literature there are different proposed to solve the above mentioned problem. In a pure topological Chern-Simons theory, Ferrari et al. [77] introduced a non-trivial kernel in the Langevin equation. Other approach was developed by the Menezes et al. [28]. These authors showed that using a non-Markovian Langevin equation with a colored random noise, the convergence problem can be solved. These authors proved that it is possible to obtain convergence toward equilibrium even with an imaginary Chern-Simons coefficient. We conclude saying that several alternative methods have been proposes to deal with interesting physical systems where the Euclidean actio is complex. These methods do not suggest any general way of solve the particular difficulties that arise in each situation.

The program of using the complex Langevin equation for an investigation of quantum field theories in Minkowski spacetime is not yet established and is still under discussion. Its clear that until the problem that we discussed above is solved, one can not say for sure that the implementation of the program of stochastic stochastic quantization in non-static pseudo-Riemannian manifolds can be successfully implemented. 


\section{Acknowlegements}

We would like to thank S. Joffily and G. Flores-Hidalgo for enlightening discussions. This paper was supported by Conselho Nacional de Desenvolvimento Cientifico e Tecnológico do Brazil $(\mathrm{CNPq})$.

\section{A The Kruskal extension of the Schwarzschild spacetime.}

The spacetime of a spherically symmetric non-rotating and eletrically neutral body is described by the Schwarzschild metric

$$
d s^{2}=-\left(1-\frac{2 M}{r}\right) d t^{2}+\left(1-\frac{2 M}{r}\right)^{-1} d r^{2}+r^{2} d \Omega^{2}
$$

where $d \Omega^{2}=d \theta^{2}+\sin ^{2} \theta d \phi$ is the metric of the unit two-sphere. The coordinate system $(t, r, \theta, \phi)$ provides a frame in which the metric components are time-independent. Since, it is possible to find a Killing vector field which is hypersurface orthogonal to the family of spacelike hypersurfaces $t=c t e$, the Schwarzschild solution is also static. The line element given by Eq.(A.1) describes the external gravitational field generated by any spherical mass, whetever its radius [78]. This includes several interesting situations, as a spherically symmetric star which undergoes radial pulsations or ever a radial spherically symmetric gravitational collapse. In the extreme case of a complete gravitational collapse, we have to consider the Schwarzschild line element as an empty spacetime solution for all values of $\mathrm{r}$. Since the line element has two singularities, one at $r=0$ and another at $r=2 M$, it represents only one of the patches $0<r<2 M$ or $2 M<r<\infty$. It is not difficult to show that although the Schwarzschild line element is singular at $r=2 M$, all the invariants constructed with the Riemann tensor and its contractions are well-behaved at $r=2 M$, and this point is a singularity due to an inappropriate choice of coordinates.

On the other hand, choosing the Schwarzschild line element to describes the patch $0<r<2 M$,

we have that the curvature scalar diverges at $r=0$, since we have $R_{\mu \nu \rho \sigma} R^{\mu \nu \rho \sigma}=\frac{48 M^{2}}{r^{6}}$. Therefore this point is a real spacetime singularity. Thus, although it is not possible to extend the spacetime across the singularity, it is posssible to analytically continue the manifold from $r>2 M$ to the region $r<2 M,(r \neq 0)$. The maximal extension of the manifold described by the Schwarzschild line element with $2 M<r<\infty$ was obtained by Kruskal.

From the condition for null geodesics it is possible to show that the radial null geodesics in the Schwarzschild spacetime are given by

$$
\left(\frac{d t}{d r}\right)^{2}=\left(\frac{r}{r-2 M}\right)^{2},
$$


and allow us to define $r^{*}$ as

$$
r^{*}=\int \frac{d r}{\left(1-\frac{2 M}{r}\right)}=r+2 M \ln \left(\frac{r}{2 M}-1\right),
$$

and it is clear that the radial geodesics must satisfy $t=r^{*}+$ cte or $t=-r^{*}+$ cte.

The next step is to define the null coordinates $u$ and $v$, where $u=t-r^{*}$ and $v=t+r^{*}$. Obviously, it is possible to write the Schwarzschild line element in terms of the null coordinates $u$ and $v$, and we have

$$
d s^{2}=-\frac{2 M e^{-\frac{r}{2 M}}}{r} e^{\frac{(v-u)}{4 M}} d u d v+r^{2} d \Omega^{2}
$$

where the metric of the unit two-sphere is inalterated. To go further, let us define new coordinates $U$ and $V$, where

$$
U=\exp \left(\frac{u}{4 M}\right)
$$

and

$$
V=\exp \left(\frac{v}{4 M}\right)
$$

Using the coordinates $U$ and $V$, the metric can be written as

$$
d s^{2}=-\frac{32 M^{3} e^{-\frac{r}{2 M}}}{r} d U d V+r^{2} d \Omega^{2} .
$$

The value $r=2 M$ is no more a singularity, since corresponds to $U=0$ or $V=0$. Finally, to obtain the Schwarzschild metric in the Kruskal form we have only to define the coordinates $T$ and $X$, by choosing $T=\frac{1}{2}(U+V)$ and $X=\frac{1}{2}(V-U)$. The final form of the Schwarzschild metric in terms of the Kruskal coordinates $(T, X, \theta, \phi)$ is

$$
d s^{2}=\frac{32 M^{3} e^{-\frac{r}{2 M}}}{r}\left(-d T^{2}+d X^{2}\right)+r^{2} d \Omega^{2} .
$$

Note that the coordinate transformation between the original coordinates $(t, r)$ and the Kruskal coordinates $(T, X)$ is given by

$$
X^{2}-T^{2}=\left(\frac{r}{2 M}-1\right) \exp \left(\frac{r}{2 M}\right)
$$

and

$$
t=4 M \tanh ^{-1}\left(\frac{T}{X}\right)
$$


Therefore, we show how it is possible to analytically continue the manifold from $r>2 M$ to the region $r<2 M$, and the event horizon, a sphere of radius $r=2 M$ is only a coordinate singularity, which can be removed by a suitable coordinate transformation. Althought the apparent singularity at the horizon has disappeared, there are true singularities in the Kruskal extension of the Schwarzschild spacetime. The physical singularity at $r=0$ corresponds to the values $X=$ $\sqrt{T^{2}-1}$ and $X=-\sqrt{T^{2}-1}$. The original Schwarzschild solution for $r>2 M$ corresponds to the region where observers can obtain information. Since there are two event horizon, the future event horizon and the past event horizon, the Kruskal spacetime defines two outer asymptotically flat regions and also two regions inside the event horizon, bounded by the past and future singularities. The black-hole is the maximal analytic extension of the vacuum Schwarzschild solution. It is well known that close to the horizon, the Schwarzschild coordinates $t$ and $r$ behaves as Rindler's spacetime coordinates.

\section{References}

[1] G. Parisi and Y. S. Wu, Sci. Sin. 24, 483 (1981).

[2] P. Damgaard and H. Heiffel, "Stochastic Quantization", World Scientific (1988).

[3] M. Namiki, Prog. Theor. Phys. Suppl. 111, 1 (1993).

[4] B. Sakita, "Quantum Theory of Many Variable Systems and Fields", World Scientific Publishing (1985).

[5] P. Damgaard and H. Heiffel, Phys. Rep. 152, 227 (1987).

[6] H. Rumpf, Phys. Rev. D33, 942 (1986).

[7] M. B. Halpern, Phys. Lett. B185, 111 (1987).

[8] M. B. Halpern, Ann. Phys. 178, 272 (1987).

[9] H. S. Chan and M. B. Halpern, Z. Phys. C36, 669 (1987).

[10] H. Rumpf, Prog. Theor. Phys. Suppl. 111, 63 (1993).

[11] H. Huffel and H. Rumpf, Phys. Lett. 148B, 104 (1984).

[12] E. Gozzi, Phys. Lett. 150B, 119 (1985). 
[13] M. Kac and J. Logan, "Fluctuations", in "Fluctuation Phenomena", E. W. Montroll and J. L. Lebowitz (Editors), North Holland Publishing Company (1974).

[14] R. Zwanzig, "Non Equilibrium Statistical Mechanics", Oxford University Press, New York (2001).

[15] R. Kubo, M Toda and N. Hashitsume, "Statistical Physics", Springer Verlag, Heidelberg (1991).

[16] G. Parisi and N. Sourlas, Nucl. Phys. B 206, 32 (1982).

[17] E. Floratos and J. Iliopoulos, Nucl. Phys. B 214, 392 (1983).

[18] W. Grimus, Z. Phys. C 18 (1983) 129.

[19] T. Fukai, H. Nakazato, J. Ohba, K. Okano and Y. Yamanaka, Prog. Theor. Phys. 69, 1600 (1983).

[20] P. H. Damgaard and K. Tsokos, Nucl. Phys. B 235, 75 (1984).

[21] P. H. Damgaard, Prog. Theor. Phys. Suppl. 111, 43 (1993).

[22] R. F. Fox, J. Stat. Phys. 16, 259 (1977).

[23] R. F. Fox, J. Math. Phys. 18, 2331 (1977).

[24] J. D. Breit, S. Gupta and A. Zaks, Nucl. Phys. B 233, 61 (1984).

[25] Z. Bern, Nucl. Phys. B 251, 633 (1985).

[26] J. Alfaro, Nucl. Phys. B 253, 464 (1985).

[27] R. Iengo and S. Pugneti, Nucl. Phys. B 300, 128 (1988).

[28] G. Menezes and N. F. Svaiter, Jour. of Math. Phys. 47, 073507 (2006).

[29] G. Menezes and N. F. Svaiter, Physica A374, 617 (2007).

[30] D. Zwanziger, Nucl. Phys. B 192, 259 (1981).

[31] Z. Bern and M. B. Halpern, Phys. Rev. D 33, 1184 (1986).

[32] J. Sakamoto, Prog. Theor. Phys. 76, 966 (1986). 
[33] Z. Bern, M. B. Halpern, L. Sadun and C. Taubes, Phys. Lett. B 165, 151 (1985).

[34] Z. Bern, M. B. Halpern, L. Sadun and C. Taubes, Nucl. Phys. B 284, 1 (1987).

[35] Z. Bern, M. B. Halpern, L. Sadun and C. Taubes, Nucl. Phys. B 284, 35 (1987).

[36] M. B. Halpern, Prog. Theor. Phys. Suppl. 111, 163 (1993).

[37] A. S. Eddington, "The Mathematical Theory of Relativity", Chelsia Publishing Company, New York, (1975).

[38] W. Rindler, Am. J. Phys. 34, 1117 (1966).

[39] S. A. Fulling, Ph-D thesis, "Scalar Quantum Field Thory in a Closed Universe of Constant Curvature", Princeton University (1972).

[40] S. A. Fulling, Phys. Rev. D10, 2850 (1973).

[41] P. W. Sciama, P. Candelas and D. Deutsch, Adv. of Phys. 30, 327 (1981).

[42] W.-H Huang, Phys. Rev. D46, 2560 (1992).

[43] N. G. van Kampen, Jour. Stat. Phys. 44, 1 (1986).

[44] N. D. Birrel and P. C. W. Davies, "Quantum Fields in Curved space", Cambridge University Press (1982).

[45] A. A. Grib, S. G. Mamayev and V. M. Mostepanenko Vacuum Quantum Effects in Strong Fields, Friedman Laboratory Publishing, St Petesburg (1994).

[46] M. Bander and C. I. Itzykson, Rev. Mod. Phys., 38, 346 (1966).

[47] A. P. Prudnikov, Yu. A. Brychkov, O. I. Marichev, "Integrals and series", Vol. 1 and 2, Gordon and Breach Science Publishers (1986).

[48] I. S. Gradshteyn and I. M. Ryshik, Table of Integrals and Products, edited by A. Jeffrey, Academic Press, Inc., New York (1980).

[49] H. Bateman, "Tables of Integral Transformations", Vol.1, California Institute of Technology (1954).

[50] N. D. Birrel, "Quantum Gravity 2", edited by C. J. Isham, R. Penrose and D. W. Sciama, Oxford University Press (1981). 
[51] T. S. Bunch, Ann. Phys., 131, 118 (1981).

[52] R. Brunetti and K. Fredenhagen, "Interacting Quantum Fields in Curved Space: renormalizability of $\varphi^{4} "$, gr-qc/9701048.

[53] M. D. Kruskal. Phys. Rev. 119, 1743 (1960).

[54] R. Wald, "Quantum Field Theory in Curved Spacetie and Black Hole Thermodynamics", University of Chicago Press, Chicago (1994).

[55] A. Strominger, "Lectures on Black Holes", Le Houches, "Fluctuating Geometries in Statistical Mechanics and Field Theory", F. David, P. Ginsparg and J. Zinn-Justin (editors), Elsevier Science B.V. (1994).

[56] R. Brout, S. Massar, R. Parentani and Ph. Spindel, Phys. Rep. 260, 329 (1995).

[57] L.H. Ford, "Quantum Field Theory in Curved Spacetime", gr-qc/9707062 (1997).

[58] T. Padmanabhan, "Gravity and the Thermodnamic of Horizon, gr-qc/0311036 (2003).

[59] S. M. Christensen and M. J. Duff, Nucl. Phys. B146, 1178 (1978).

[60] P. Candelas and D.J. Raine, J. Math. Phys. 17, 2101 (1976).

[61] G. W. Unruh, Phys. Rev. D14, 870 (1976).

[62] S. A. Fulling and S. N. M. Ruijsenaars, Phys. Rep. 152, 135 (1987).

[63] C. A. D. Zarro, M. A. thesis, "Teoria Quântica de Campos na Presença de Horizonte de Eventos", Centro Brasileiro de Pesquisas Físicas - CBPF (2006).

[64] G. 't Hooft, Nucl. Phys. B256, 727 (1985).

[65] G. 't Hooft, Acta Phys. Pol. B19, 187 (1988).

[66] C. D. Fosco and N. F. Svaiter, Jour. Math. Phys. 42, 5185 (2001).

[67] M. I. Caicedo and N. F. Svaiter, Jour. Math. Phys. 45, 179 (2004).

[68] N. F. Svaiter, Jour. Math. Phys. 45, 4524 (2004).

[69] M. J. Aparicio Alcalde, G. H. Flores and N. F. Svaiter, Jour. Math. Phys. 47, 052303 (2006). 
[70] H. Huffel and P. V. Landshoff, Nucl. Phys. B260, 545 (1985).

[71] D. J. Callaway, F. Cooper, J. R. Klauder and H. A. Rose, Nucl. Phys. B262, 19 (1985).

[72] G. Parisi, Phys. Lett. B131, 393 (1983)

[73] J. R. Klauder and W. P. Peterson, J. Stat. Phys. 39, 53 (1985).

[74] J. R. Klauder, Phys. Rev. A29, 2036 (1984).

[75] J. Ambjorn and S. K. Yang, Phys. Lett. 165, 140 (1985).

[76] H. Okamoto, K. Okano. L. Schulke and S. Tanaka, Nucl. Phys. B342, 684 (1989).

[77] F. Ferrari and H. Hufel, Phys. Lett. B261, 47 (1991).

[78] G. Birkhoff, "Relativity and Modern Physics", Havard University Press, Cambridge (1923). 